\title{
Digenean-gastropod host associations inform on aspects of specific immunity in snails
}

C.M. Adema ${ }^{\mathrm{a},{ }^{*},}$, E.S. Loker ${ }^{\mathrm{a}}$

${ }^{a}$ Center for Evolutionary and Theoretical Immunology, Department of Biology, University of New Mexico, Albuquerque, NM 87131, USA.

coenadem@unm.edu,esloker@unm.edu.

*Corresponding author

C.M. Adema

Center for Evolutionary and Theoretical Immunology,

Department of Biology,

University of New Mexico,

Albuquerque, NM 87131, USA

coenadem@unm.edu

$+1(505) 2772743$

(C) 2014. This manuscript version is made available under the Elsevier user license http://www.elsevier.com/open-access/userlicense/1.0/ 


\begin{abstract}
Gastropod immunology is informed importantly by the study of the frequent encounters snails endure with digeneans (digenetic trematodes). One of the hallmarks of gastropod-digenean associations is their specificity: any particular digenean parasite species is transmitted by a limited subset of snail taxa. We discuss the nature of this specificity, including its immunological basis. We then review studies of the model gastropod Biomphalaria glabrata indicating that the baseline responses of snails to digeneans can be elevated in a specific manner. Studies incorporating molecular and functional approaches are then highlighted, and are further suggestive of the capacity for specific gastropod immune responses. These studies have led to the compatibility polymorphism hypothesis: the interactions between diversified fibrinogen-related proteins (FREPs) and diverse carbohydrate-decorated polymorphic parasite antigens determine recognition and trigger specific immunity. Complex glycan structures are also likely to play a role in the host specificity typifying snail-digenean interactions. We conclude by noting the dynamic and consequential interactions between snails and digeneans can be considered as drivers of diversification of digenean parasites and in the development and maintenance of specific immunity in gastropods.
\end{abstract}




\section{Keywords}

Biomphalaria glabrata: host specificity: host-parasite interactions; immune priming; acquired immunity; schistosomiasis 


\section{Contents:}

1. Introduction

2. Host specificity of digenean-snail interactions: an indicator of the underlying innate capacity for specificity of the gastropod immune system

2.1 Characteristics and variations of digenean-snail specificity

2.2 Gastropod immunobiology is a determinant of host-specificity

3. Acquired resistance of snails to digenean infection: another indication of the capacity for specificity

4. From snails to genes and immunity

5. What is the mechanistic basis of digenean specificity for snails?

6. Can gastropod immune specificity be relaxed or modified?

7. Evolutionary considerations

Acknowledgments

References 


\section{Introduction}

The emphasis of this review is on the specific nature of the gastropod immune response. After considering various ways to define "specificity" that ended up seeming arbitrary or less relevant to the biology of gastropods themselves, we decided to use as our operational indicator of specificity something provided by one of the most relevant, and certainly one of the betterstudied groups of dedicated gastropod parasites, the digeneans (also known as flukes or digenetic trematodes). For our purposes here, specific immunity of snails is considered to be the capability of their internal defenses to recognize a particular taxon or group of closely-related taxa of digeneans and effect immune-mediated elimination, either innately, or in a manner that is improved after a previous exposure to the same or related digeneans. Given that the systematics of digeneans is steadily improving, and the group is remarkably diverse, we can think of them as being relatively well-defined, diverse and relevant tools to probe the nature of gastropod immunity. By no means do we intend to imply that the only manifestations of specificity in gastropod defenses apply to digeneans, nor that snail responses to digeneans fully represent the entire spectrum of snail immune capabilities.

We readily acknowledge that the extent to which invertebrate defense responses are specific, and the extent to which invertebrates are anatomically equipped (e.g. lack of lymphocytic tissues, clonal expansion) to mount specific responses that can be meaningfully enhanced upon re-exposures, are long-standing issues of debate in studies of comparative or evolutionary immunology (Hauton and Smith, 2007; Klein, 1989; Loker et al., 2004). Many recent studies suggest the diversity of molecules available to various groups of invertebrates to counter pathogens and parasites may be much higher than previously considered (e.g. Buckley and Rast, 2012; Ghosh et al., 2011), and that at least some invertebrates can tailor their immune responses to the pathogens encountered. The fruit fly Drosophila melanogaster employs various non-self recognition receptors that distinguish Gram (-) from Gram (+) bacteria or other pathogens, and activate different signaling pathways that generate transcriptional profiles to 
emphasize defense factors with greatest impact on the pathogens encountered (Ferrandon et al., 2007; Hoffmann and Reichart, 2002; Kraaijeveld et al., 2012). Moreover, previous encounters that result in immune elimination of pathogens lead to immune priming; fruit flies retain increased immune resistance to specific pathogens for about a week (Christofi and Apidianakis, 2013, Pham et al., 2007). Many of the authoritative papers in the field derived from studies of deuterostome invertebrates or of ecdysozoan protostomes, with studies of Lophotrochozoa, the other major branch of protostome invertebrates (Erwin et al., 2011), tending to lag behind.

Here we attempt to make the case that some of the hallmark features of more sophisticated invertebrate defenses, including mounting specific responses that can be elevated to heightened levels, are exemplified by gastropod molluscs, particularly in their interactions with members of another prominent lophotrochozoan phylum, the Platyhelminthes, specifically the Digenea. Both gastropods and digeneans are species-rich groups. A fundamental commitment of digeneans to a highly invasive mode of development in gastropods was made long ago, and has been largely retained subsequently. The nature of the digenean development program within gastropods is one that is remarkable for the extent to which it involves proliferative asexual development, with dire fitness consequences for snails. We argue that it is precisely within such a long-standing, biotically complex interaction, one with high fitness stakes and diverse hosts and parasites involved, that one might expect to see some of the most sophisticated aspects of parasite offense and host defense played out.

This review first discusses the nature and range of naturally occurring compatible gastropod-digenean parasite associations - what is often referred to as snail-digenean host specificity - as an indication of specific innate immunity in snails. We then review some of the classic experimental studies (in our view, too often neglected) that demonstrated snails can gain specific immunity, termed acquired resistance, when challenged with particular lineages of digeneans. We then consider more modern studies to interpret the molecular foundations of 
specific immunity in the snail B. glabrata. We conclude with some consideration of the potential macroevolutionary impacts of specific immune interactions between digeneans and snails.

\section{Host specificity of digenean-snail interactions: an indicator of the underlying innate}

\section{capacity for specificity of the gastropod immune system}

One of the most prominent features of gastropod biology is their involvement as first intermediate hosts in the life cycles of most of the estimated 25,000 species of digenetic trematodes (Esch et al., 2002). Miracidia of digeneans colonize snails and initiate a program of asexual reproduction that yields sporocyst and/or redia stages, and culminates in the production of cercariae, usually after 4-6 weeks. Infection heavily impacts snail fitness because digeneans generally castrate their hosts, yet infected snails may persist in releasing cercariae for months and sometimes years. All the while, the digenean larvae lie in intimate contact with the snail host's tissues.

The associations between digeneans and their snail hosts are specific, and digeneans are said to be host specific with respect to their snail hosts (see Adema and Loker, 1997 for general discussion and examples, and section 2.1 below). Digenea are highly specialized parasites; almost the entire lineage has committed to molluscs (mostly gastropods) as first intermediate hosts. The capacity of any given digenean to infect snails is not limitless across all snail species; distinctive patterns of host use have emerged. Such characteristics of digenean-snail specificity can help probe and understand the nature, including specificity, of the gastropod immune system.

\subsection{Characteristics and variations of digenean-snail specificity}

Although the literature has been characterized as implying that a particular digenean species can develop in only one species of snail (Donald et al., 2004), digenean-snail host specificity is not so simply summarized. Extensive studies of specificities of the medically important schistosomes (see Basch, 1976; Brown, 2005 for good historical reviews) indicate that 
Schistosoma mansoni only infects snails of a single genus, Biomphalaria (family Planorbidae). At least 18 of the circa 34 species of Biomphalaria are susceptible to this trematode (DeJong et al., 2001). Specificity can in some cases be even more pronounced, such that some strains of species like Biomphalaria glabrata are susceptible to S. mansoni, whereas others are resistant (Richards et al., 1992), yet other B.glabrata strains are susceptible to one strain of S. mansoni and resistant to another S. mansoni strain (Lie et al., 1982). Species of Helisoma, the sister genus of Biomphalaria, are uniformly refractory to S. mansoni infection. Many other planorbid snails from different genera have been exposed to $S$. mansoni but consistently fail to take the infection (see Sullivan and Yeung, 2011). Similarly, Schistosoma haematobium normally develops only in certain species of Bulinus snails (Rollinson et al., 2001). This "rule" regarding host specificity for schistosomes is rarely violated. Planorbarius metidjensis, not a particularly close relative of Biomphalaria or Bulinus, can be infected by both S. haematobium (Kechemir and Combes, 1982) and S. mansoni (Barbosa et al., 1959) but this snail is probably of little or no significance in natural transmission. Schistosoma haematobium is unable to infect Biomphalaria just as $S$. mansoni is unable to infect Bulinus.

The relationship between S. mansoni and Biomphalaria provides further insight regarding host specificity. After the genus Biomphalaria first diversified in the Americas, a snail similar to modern-day B. glabrata is believed to have colonized Africa and to have given rise to the African Biomphalaria species about 1.4-4.3 million years ago (DeJong et al., 2001). Strong incompatibility or resistance among the 12 African species of Biomphalaria to S. mansoni are rarely reported, although Biomphalaria alexandrina seems to be relatively less compatible to $S$. mansoni from various sources (Frandsen, 1979). The general compatibility between African Biomphalaria and S. mansoni is not surprising given this parasite most probably evolved on the African continent. By contrast, among the 17 South American Biomphalaria species, 8 species are generally refractory. Although South America is where Biomphalaria first diversified, $S$. mansoni did not encounter Biomphalaria there until 400-600 years ago, upon the arrival of 
infected Africans. In the absence of S. mansoni, some - but not all - lineages of Biomphalaria in the Americas have evolved and have retained (and shared with African Biomphalaria), distinctive properties that allow exploitation by $S$. mansoni. Once these biological features were established, even without continuous selective pressure from S. mansoni, they have been retained by those snails such that they can still support $S$. mansoni infections. Although there is a tendency for sympatric combinations of S. mansoni and Biomphalaria to be more compatible than allopatric combinations, often $S$. mansoni isolates from one geographic area readily infect Biomphalaria snails from distant areas (Kagan and Geiger, 1965, Frandsen, 1979).

Not surprisingly, the degree of host specificity exhibited by different digenean species is variable, even including among closely related species. The liver fluke Fasciola hepatica is able to develop in some, but not all, representatives of the following snail genera: Galba, Lymnaea, Omphiscola, Stagnicola, Pseudosuccinea, and Radix (Caron et al., 2014). This hardly seems very specific, but all of these genera are found within a single molluscan family, the Lymnaeidae. Echinostoma paraensei can develop in members of three different pulmonate families (Maldonado et al., 2001). Whereas Clonorchis sinensis is able to infect snails of five caenogastropod families, the closely-related Opisthorchis viverrini infects only one of these families, the Bithyniidae (Petney et al., 2013).

Although fewer experimental studies have been done with marine snails, specificity with respect to digeneans seems to apply here too. For example, for genetically-defined (characterization of $16 \mathrm{~S}$ and ITS2 sequences) opecoelid trematodes in trochid snails, two of the digenean lineages studied were found in only one species of snail host, while two other lineages were found to be more general, one infecting two and one infecting three snail species each (Donald et al., 2004). The fluke Dicrocoelium dendriticum parasitizes land snails, infecting representatives of seven gastropod families, all within the clade Stylommatophora (MangaGonzalez et al., 2001). Similarly, species of Brachylaima are also able to infect land snails of 
multiple families of Stylommatophora (Butcher and Grove, 2001). This suggests that the degree of digenean host specificity in stylommatophoran snails may be lower. Even here, however, there are definite limits to the range of snails that can be colonized by one digenean species. We know of no unambiguous examples, where a single genetically-defined digenean parasite can colonize and successfully reproduce asexually within both caenogastropods and pulmonate snails.

\subsection{Gastropod immunobiology is a determinant of host-specificity}

In several instances, a good case has been made that miracidia exert behavioral discrimination such that they penetrate appropriate snail hosts and avoid penetration of inappropriate hosts (Haas, 2003; Sullivan and Yeung, 2011). Yet there are many cases known in which miracidia readily attach to and penetrate inappropriate hosts that are not favorable to their subsequent development (see Sapp and Loker, 2000a and references therein), so much so that the use of incompatible "decoy" snails to harmlessly exhaust or absorb schistosome miracidia has been mentioned repeatedly as a means to control schistosomiasis (Chernin and Perelstein, 1971; Combes and Moné, 1987; Johnson et al., 2009). It seems unlikely that pre-penetration discriminatory behavior is the whole explanation underlying host specificity, but it is one that remains understudied and may be particularly relevant when digenean miracidia encounter snails that are more distantly removed phylogenetically from their normal snail hosts. We return to a discussion of the role of host-penetration behavior in section 5 because some of the factors involved in both behavioral and immunological discrimination, namely glycan moieties associated with glycoproteins, play a role in both phenomena.

Consider too the evidence that has shown that the presence of cells from a line originally derived from embryos of $B$. glabrata will support enhanced growth and development of sporocysts of S. mansoni that normally develop in B. glabrata, but also favor development of sporocysts of $S$. japonicum that develop in distantly related caenogastropods of the family Pomatiopsidae, and also of sporocysts of Fascioloides magna, an unrelated parasite that develops 
in a different family of pulmonates, the Lymnaeidae (Coustau and Yoshino, 2000). This indicates that cells of a non-natural host snail species can support early stages of digenean development, implying that reasons pertaining to specific nutritive requirements by themselves seem to be insufficient in all cases to account for the host specificity shown by each of the trematode species mentioned above..

In some cases, miracidia attempt to penetrate inappropriate snail species and even though they may eventually swim away and survive the encounter, may be "exhausted" and unable to penetrate other snails (Combes and Moné, 1987). This may merely reflect a depletion of energy reserves or of penetration enzymes so may not have an immunological basis, but does not rule out the possibility that distinctive host components can disable parasite penetration enzymes or otherwise sabotage the penetration effort. The role of the epithelial barrier as a significant component of defense against digenean infection is exemplified by studies of cyclocoelid flukes, which have a unique strategy for entering the molluscan host. Instead of penetrating the snail's epithelium, the miracidia of cyclocoelids attach to the epithelium and then inject a single redia through the epithelium into the tissues. It is noteworthy that cyclocoelid species like Cyclocoelum mutabile have an unusually broad spectrum of potential snail hosts, with members of at least three snail families serving this role (McKindsey and McLaughlin, 1995). The same is true of other cyclocoelid species. This implies the epithelial barrier is formidable for species that have to burrow their way through it.

Penetration of miracidia into non-host snail species occurs in some cases and is often followed by encapsulation of the miracidia (Sudds, 1960), strongly suggestive of the direct involvement of the gastropod immune system in recognizing and eliminating maladapted parasites. In an example involving bivalve hosts, hemiuroid sporocysts were observed to be encapsulated in an incompatible host, but not in a compatible host. The difference was ascribed to greater similarity between the surface carbohydrates of the sporocysts and hemocytes of the susceptible host than with sporocysts and hemocytes of the incompatible host species (Kawasaki 
et al., 2013). Miracidia that are experimentally injected into snails are often encapsulated in incompatible hosts, but can survive and develop in compatible hosts (Heyneman, 1966; Nunez and De Jong-Brink, 1997; Sullivan and Yeung, 2011), implying that recognition and destruction by immune system components can occur once within the internal milieu of the snail. Nunez and DeJong-Brink (1997) noted that secretory-excretory products derived from sporocysts of Trichobilharzia ocellata had a suppressive effect on bacterial clearance by hemocytes of the host snail Lymnaea stagnalis, but had no comparable effect on the hemocytes of the non-host species Planorbis corneus. This indicates that, at least in this host-parasite pair, suppressive effects of digenean larvae are specific in their action on hemocytes of compatible host species.

The involvement of humoral components of the immune system in host specificity is suggested by studies in which sporocysts and/or rediae from four different digenean species were placed in plasma from 5 different snails species, including incompatible and compatible hosts. In no case was plasma from a compatible host found to result in damage to a sporocyst or redia, whereas in 8 of 16 incompatible combinations examined, snail plasma had a significant negative effect on sporocyst condition (causing rounding, blebbing, or death). Plasma from lymnaeid snails was generally harmful to parasites of planorbid snails, and in 2 of 4 cases, planorbid plasma was harmful for parasites of lymnaeid snails. Toxicity could not be ascribed to differences in osmolality (Sapp and Loker, 2000a). In the same experimental set up, there was no overall pattern to suggest that hemocytes were more likely to bind incompatible than compatible parasites in a one hour in vitro contact assay (Sapp and Loker, 2000b). Sudds (1960) noted that in some cases when miracidia succeeded in penetrating incompatible hosts, sporocysts were not surrounded by hemocytes but appeared to degenerate with time, also indicative of a role for humoral factors. Finally, using approaches akin to vaccination termed immune priming, a host species normally susceptible to a particular parasite can be rendered resistant, suggesting that stimulation of the immune response can change the host status of a snail (see more discussion in section 3). 
For the reasons stated above, admittedly far from being a clear cut case, it seems probable to us that the phenomenon of host specificity in digenean-snail associations topic is not independent from the discriminatory capability of the snail immune system. This suggests that significant differences exist among various groups of snails in their ability to discriminate among different digenean species, in turn implying that in the face of very relevant pathogens (digeneans are common and have severe fitness consequences), snail defense responses show specificity. Whereas we view host specificity as an expression of the innate specificity of gastropod defenses, below we highlight the phenomenon of acquired resistance as another manifestation of specificity of the gastropod immune system.

\section{Acquired resistance of snails to digenean infection: another indication of the capacity for specific immunity}

The studies discussed in this section largely focus on one snail species, Biomphalaria glabrata, a major intermediate host of Schistosoma mansoni in the New World. This species, subject of a soon to be completed genome study, has become the most extensively used model host for all kinds of schistosome-snail investigations, including studies of acquired resistance. Studies of this phenomenon have included several other species of trematodes, most of them in the genus Echinostoma that also employ B. glabrata as a first intermediate host. Echinostome miracidia penetrate snails and transform into sporocysts that migrate to the heart and there give rise to mother rediae. Mother rediae leave the sporocyst and give rise to daughter rediae in which cercariae later develop, exit, and leave the snail.

This story begins with a remarkable set of studies performed by Lie, Heyneman and coworkers in the mid 1970's to the early 1980's (Lie, 1982; Lie et al., 1987), that continue to provide foundational insights into the capability of snails to acquire specific resistance to relevant parasites like digeneans. It was initially observed that a small percentage of snails among labmaintained B. glabrata was naturally resistant to experimental infections with miracidia of 
Echinostoma lindoense. Sporocysts migrated to the heart as usual, but then were encapsulated and destroyed within 2-5 days. Thirty-three of 35 snails that had eliminated all parasites from the initial infection in this manner responded faster to E. lindoense sporocysts when exposed a second time (using 100 miracidia at between 4 and 14 days after resolution of the first infection). Challenge sporocysts did not reach the heart but rather were encapsulated close to their penetration sites in the headfoot of sensitized snails and destroyed within 2 days (Lie and Heyneman, 1975).

The acquired resistance provoked by sensitization with $E$. lindoense had a considerable degree of immune specificity, providing partial resistance to infection by related parasites (Echinostoma liei and E. paraensei) in a small proportion of sensitized B. glabrata. No protection was afforded against more distantly related digeneans such as S. mansoni and Paraphystomum segregatum (Lie and Heyneman, 1975). Sensitization was also achieved through the use of radiation-attenuated E. lindoense miracidia that migrate to the heart of B. glabrata but die shortly thereafter. The subsequent encapsulation and elimination of all parasites yielded acquired resistance in all experimentally-exposed snails, with the same specificity as evoked in naturally resistant B. glabrata (Lie et al., 1975). A second re-exposure (3-4 days after a first reexposure at 5-8 days post-sensitization with attenuated miracidia) further accelerated the rate of parasite elimination in the head foot tissues to within 30 hours after infection. Evaluation of histological sections showed anti-parasite encapsulations in both a first sensitizing exposure and after repeated infections (Lie and Heyneman, 1976). Lie et al. (1976) concluded that hemocyte producing tissues (including the APO or amoebocyte-producing organ, and peripheral foci of hemocyte production) displayed increased activity during formation of major hemocyte encapsulations, and this likely contributes additional hemocytes to effect natural or acquired resistance. A comparative study showed that each of four B. glabrata strains were able to develop acquired resistance after exposure to irradiated miracidia of various echinostome species although 
the strength of resistance varied for specific combinations of snail strain with each of the three species of echinostomes tested (Lie and Heyneman, 1979).

Induction of acquired resistance in B. glabrata to S. mansoni proved elusive with several B. glabrata strains until sensitization with attenuated miracidia yielded a modest (yet significant) degree of protection against challenge infection in juvenile snails of the 442132 strain of $B$. glabrata. This strain, unfortunately no longer available, displayed juvenile susceptibility combined with adult resistance to $S$. mansoni (Lie et al., 1983 and references therein). A recent study involving genetically characterized and matched strains of S. mansoni and B. glabrata again showed that exposure to irradiated miracidia did not yield acquired resistance (Portela et al., 2013). However, this study did report that previously established (primary) S. mansoni infection protected B. glabrata against secondary infections with a homologous parasite genotype. This observation, similar to that of Sire et al. (1998), awaits further clarification as to the underlying mechanisms responsible for failure of the challenge infection. Additionally, a degree of immune priming was achieved experimentally by Portela et al. (2013) by first injecting B. glabrata with miracidial extracts. Partial protection against challenge infection (10 miracidia administered 15 days after sensitization) from a homologous schistosome strain was observed (prevalence of 67\%, significantly different versus $90 \%$ for the sham-injected control).

Taken together, both older and more recent results suggest it is not very likely that specific acquired immunity imposes a significant restriction to $S$. mansoni transmission by $B$. glabrata under natural conditions. Indeed, experiments by Kagan and Geiger (1965) and File (1975) showed that when B. glabrata already shedding S. mansoni cercariae were later exposed to additional miracidia, some of these miracidia were able to develop and produce cercariae. Microsatellite and Southern blot studies have shown that field-collected Biomphalaria snails, including B. glabrata, are not uncommonly shedding cercariae of multiple genotypes, with as many as 8 genotypes reported from one snail (Minchella et al., 1995; Steinauer et al., 2008). The overall results suggest that the underlying interactions of echinostomes and schistosomes with $B$. 
glabrata differ, with it easier to evoke acquired resistance in the case of echinostomes, possibly because $B$. glabrata is more prone to develop age-related resistance to echinostomes than to schistosomes. Lie, Heyneman and coworkers did not have access to molecular tools to analyze the mechanistic basis for their observations, but the robust experimental framework they established provided an ideal starting point for later studies using molecular approaches to explore the underlying mechanisms, as discussed in the following section.

\section{From snails to genes and immunity.}

The prominent role of encapsulation in elimination of digenean parasites provided a good rationale to focus immunobiological studies on the functional aspects of hemocytes, the defense cells circulating in blood and tissues of snails. Hemocytes are potent mediators of cytotoxicity, that dispose over lysosomal enzymes and are able of the respiratory burst, the production and release of highly toxic reactive oxygen species (ROS) to damage and eliminate larval digeneans (e.g Adema et al., 1990; Hahn et al., 2001). To achieve specific immunity, hemocytes need to be activated to mount defense responses. Indeed, hemocytes from compatible B. glabrata fail to respond to $S$. mansoni but can be activated to engage in immune activities through soluble factors in cell-free blood fluid taken from B. glabrata strains known to be immunologically resistant to the parasite (Loker and Bayne, 1982, Vasquez and Sullivan, 2001). The mediators of non-self recognition were identified as lectins (Renwrantz, 1986), non-enzymatic proteins that function as pattern recognition receptors (PRRs) that bind repetitive carbohydrate determinants termed pathogen-associated molecular patterns (PAMPs) and signal to activate immune responses (e.g. Vasta et al., 2012). Indeed a variety of lectin-like, carbohydrate-binding activities was shown in $B$. glabrata and investigated in the context of non-self recognition and immune function towards digeneans (see van de Knaap and Loker, 1990). Infection with the echinostome E. paraensei evoked a clear and robust response in B. glabrata, consisting of increased abundance of plasma lectins that bound parasite antigens (Adema et al., 1997a). The application of then newly 
available molecular techniques resulted in the molecular characterization of several of these snail lectins as fibrinogen-related proteins (FREPs: Adema et al 1997b). FREP lectins revealed a unique composition of upstream immunoglobulin sequence domains and fibrinogen-like sequence at the C-terminal. With sequence identification, snail immune function could be investigated for the first time at the level of specific gene products rather than by activity or by group of proteins of uncertain characteristics. The advent of routine automated DNA sequencing afforded gene discovery through random expressed sequence tags (ESTs) and this drove a large number of studies (an incomplete yet representative listing includes e.g. : Hanelt et al., 2008, Lockyer et al., 2007, Mitta er al., 2005, Raghavan et al, 2003) that provide identification of many additional putative immune factors of B. glabrata, (e.g Baron et al., 2013; Galinier et al., 2013; Garcia et al., 2010; Guillou et al., 2004; Yoshino et al., 2008), revealing potential immune mechanism that could be studied further for a better understanding of snail immunity. This trend continues with the imminent characterization of the B. glabrata genome.

Genomic level studies have enhanced understanding of ROS production by B. glabrata hemocytes as a particular immune mechanism with a role in specific immunity. A comparison of two compatible snail-parasite combinations revealed a close matching of the levels for ROS generation by $B$. glabrata hemocytes and the expression in $S$. mansoni of protective enzymes that detoxify ROS (Moné et al., 2011). Specific immunity to $S$. mansoni in lab-maintained $B$. glabrata populations correlated with high expression of a particular allele of $\mathrm{Cu} / \mathrm{ZnSOD}$ associated with elevated ROS production by hemocytes of resistant versus susceptible B. glabrata (Bonner et al., 2012). Thus the SOD1B allele provides a marker for specific resistance in $B$.

glabrata to S. mansoni. The authors, however, caution that they specifically studied the interaction between PR-1 strain S. mansoni and B. glabrata derived from the 13-16-R1 lab strain, and it is not clear whether SOD1 alleles may influence resistance to other pathogens that may be encountered under natural conditions. It seems less probable that a quantatitive difference in ROS production capacity is at the origin of specific immunity of B. glabrata to digeneans in general, 
especially considering the potential of these parasites to evolve more effective countermeasures and the diversity of digeneans that can infect B. glabrata.

The publicly available expression sequence data resulting from gene discovery efforts have been used to develop microarray platforms for more comprehensive study of the immune capabilities of B. glabrata toward pathogens (Adema et al., 2010; Hanington et al., 2010a, 2010b; Lockyer et al., 2008; 2012). Such approaches have revealed differences in the hemocyte responses to $S$. mansoni of susceptible and resistant $B$. glabrata, involving functional components and indicating possible regulatory pathways that have a role in effective immunity toward pathogens. Highthroughput RNAseq capabilities, providing additional insights into relative expression of particular sequences, continue to further inform on the B. glabrata responses to pathogens. Remarkably, analysis by Deleury et al. (2012) of Illumina data suggested that defense responses from B. glabrata to bacteria and yeast include highly similar immune processes and signaling pathways but different gene sequences belonging to multigenic families are employed depending on the pathogen encountered.

FREPs are routinely identified as part of the $B$. glabrata defense response, and in particular as a major common feature of different types of resistance to digeneans, including strain-specific resistance, age-dependent resistance and acquired immunity to echinostomes (Hanington et al., 2010a, 2010b, 2012). While the exact functional mechanism remains to be resolved, the involvement of FREPs in immunity is evident by reduced resistance to digenean infections in B. glabrata snails that were subjected to FREP knockdown by RNA interference (Hanington et al., 2010b, 2012). It is highly attractive to consider a role for FREPs in non-self recognition. These lectins increase in abundance after infection and bind parasite antigens and carbohydrates in a reversible manner (Adema et al., 1997b). FREPs are encoded by extensive and diverse gene subfamilies consisting of highly related sequences that have different binding preferences and may be specialized to interact with particular pathogens (comparing the digenean E.paraensei, yeast, Gram-positive and Gram-negative bacteria; Zhang et al., 2008). Moreover, 
random somatic diversification of FREP genes through point mutations and gene conversions (Zhang et al., 2004), gives rise to highly diverse repertoires of FREPs both between and within individual B. glabrata snails, even among subsets of hemocytes (Hanington et al., 2010b). This somatic mutation is hypothesized to occur in some but not all developing hemocytes as they differentiate in hemocyte producing tissues, especially following immune stimulation of $B$. glabrata (Stout et al., 2009). The mechanism and the gene target(s) specificity of this somatic mutation remain to be characterized. Once mature, hemocytes express and release FREPs that circulate as plasma proteins. FREP diversity in an individual B. glabrata, additional to expression of germline-encoded FREP genes, continuously varies with the maturation and continuous replacement of hemocytes. The generation and expression of polymorphic FREPs extends the range of non-self recognition capability beyond the sequence diversity that is encoded in the germline, providing B. glabrata individually unique recognition capabilities toward pathogens. The baseline recognition capabilities are defined by germline FREP genes (including alleles) that provide a genetic lineage of $B$. glabrata with a standard range for predictable specific recognition and immunity to particular pathogens. This range may then be supplemented with random diversity over time, through a continuously changing repertoire of variant FREP sequences to increase the range of antigens that can be responded to. Immune priming then coincides with the time interval that hemocytes circulate to provide and maintain a particular collections of FREP variants that is available for non-self recognition before replacement hemocytes contribute newly mutated FREPs. This model fits with the notion that recognition leading to specific immunity, or lack of recognition resulting in immune compatibility, is decided at the individual level each time that a snail and digenean parasite encounter occurs, and depends on the genetic background of both partners (Basch, 1976; Théron and Coustau, 2005).

\section{What is the mechanistic basis of digenean specificity for snails?}


The answer remains elusive, but emerging from the detailed study of the S. mansoni - B. glabrata system are converging insights regarding gastropod immunology that seem to provide a compelling new way to study the phenomenon of host specificity. Several recent studies (Lehr et al., 2010; Yoshino et al., 2012, 2013) have shown that $S$. mansoni miracidia and sporocyst surface proteins and sporocyst transformation products bear glycan structures that are similar to those found expressed on plasma proteins or hemocytes of B. glabrata. This similarity adds significant additional evidence to a long-standing hypothesis (Damian, 1964) that parasites may escape immunological detection in their hosts by molecular mimicry. Further support for this idea comes from the observation that plasma proteins from susceptible strains of $B$. glabrata have a greater distribution and abundance of sharing of some glycans than plasma proteins from a schistosome resistant strain (Yoshino et al., 2012). This may in turn enable non-self detecting molecules circulating in snail plasma, including the diverse FREP plasma proteins (Zhang et al., 2004), to recognize sporocysts more readily in resistant snails. Furthermore, exposure of plasma proteins or hemocytes to sporocyst transformation products alters glycan expression, potentially interfering with the binding of plasma factors to either sporocysts or hemocytes (Yoshino et al., 2013). If plasma proteins serve as essential bridging molecules between sporocyst surfaces (or soluble sporocyst derived molecules) and hemocytes, then sporocyst transformation products could disrupt this interaction.

All of these results are basically in line with the compatibility polymorphism hypothesis (Mitta et al., 2012) that postulates that diverse glycans and associated glycoconjugates produced by schistosome sporocysts may be serving as PAMPs that are recognized by diversified fibrinogen-related proteins of FREPs. Complexes of FREPs and polymorphic mucins from $S$. mansoni have been isolated (Moné et al., 2010), and studies of FREP3 knockdown snails have yielded phenotypes more susceptible to echinostome and schistosome infection (Hanington et al., 2010b, 2012). Diversified polymorphic mucin proteins, coupled with a large potential variety of 
glycan structures with which they may be adorned (Yoshino et al., 2012), could provide a huge repertoire of antigenic diversity to be countered by diversified recognition molecules like FREPs.

The relevance of this work to the study of host specificity per se is that if different snail species, genera and families routinely display different glycan structures, then a prediction could be made that the larval stages of trematodes should bear a greater degree of glycan sharing with compatible than incompatible hosts (see too Kawasaki et al., 2013). Although there may well be other aspects of molluscan biology that require different lineages of snails to express different suites of glycans, it should not be discounted that one possible factor favoring diversification of glycan structures among different gastropod lineages is parasitism by trematodes. Here a comparative approach involving several digenean and snail species could be profitable, rather than the more standard approach of detailed study of a single digenean and snail combination.

It is clearly the case that the degree of specificity varies and, for some digenean-snail systems (some digeneans in land snails), does not appear to be rigid. One possible explanation is that this merely reflects the presence of cryptic species of trematodes, such that when their full diversity is revealed, the degree of specificity is actually greater than it presently seems. Another possibility is that not all gastropod groups bear distinctive glycan profiles such that a single trematode could match several species. Yet another possibility for broader host ranges for some digenean species is that they employ other protective mechanisms, such as interference (Lie, 1982; Loker et al., 1992). Here more overt mechanisms of damaging or altering the functional integrity of the gastropod immune system are employed and may not require the degree of epitope matching implied by molecular mimicry.

Lastly, as briefly noted in section 2.2, free-swimming miracidia of some parasites are able to differentiate between appropriate and inappropriate hosts, and their perception of glycans on glycoproteins secreted by the snails are believed to be critical to this process. Perhaps fundamentally the same system is at work both outside and inside the snail. A miracidium sensing a glycan mismatch would not engage in penetration, potentially sparing it from later attack by 
plasma proteins able to detect different glycan structures on a maladapted parasite. This possibility too would benefit from additional comparative studies employing panels of digenean and snail species.

\section{Can gastropod immune specificity be relaxed or modified?}

The host range for a particular digenean species can in some cases be extended if a non-host strain or species is first exposed to another digenean species, in what is known as facilitation, and that also been called acquired susceptibility. The presence of the facilitating parasite species is believed to diminish the immune capability of the snail such that the normally incompatible digenean can survive. Usually, the host range extensions that can be achieved in this way are modest. Snails of schistosome-resistant strains of Biomphalaria glabrata can be rendered susceptible to this parasite if first exposed to miracidia of E. paraensei (Hanington et al., 2012; Lie, 1982). Exposure to the echinostome has been shown to result in down-regulation of several components of the B. glabrata defense response (Adema et al., 2010; Hanington et al., 2010a), apparently disabling those components responsible for preventing S. mansoni development. Echinostoma paraensei is also known to have a strong effect on host hemocyte structure and function, making it harder for normally resistant snail strains to form coherent encapsulations responses around S. mansoni sporocysts (Loker et al., 1992).

With respect to extending host range to new species, prior infection of Biomphalaria orbignyi and Biomphalaria oligoza with the amphistome Zygocotyle lunata followed by exposure to $S$. mansoni enables the latter parasite to colonize these normally schistosome refractory snail species (Spatz et al., 2012). Similarly, the snail Bulinus tropicus is normally refractory to Schistosoma bovis, but can be successfully infected with this species if it is first exposed to the amphistome Calicophoron microbothrium (Southgate et al., 1989). We know of no experimentally documented examples where facilitation has extended the host range of a particular digenean species into a new genus or family of snail host. 
The schistosome Austrobilharzia terrigalensis is apparently completely dependent upon facilitation for its successful larval development in snails, as it is able to initiate infections in the snail Velacumantus australis only if the snail already harbors another trematode infection. This schistosome is somehow able to suppress the larval development of the resident trematode and eventually begins to produce its own progeny (Walker, 1979). The extent to which the host range of this species could be extended to other snail species via similar facilitation mechanisms is unknown.

There are also occasional instances when infected snails recovered from the field seem to violate the well-known normal host range for a particular trematode species. For example, as noted above, the liver fluke Fasciola hepatica can infect several but by no means all species within the family Lymnaeidae. There is one report of this species infecting Biomphalaria alexandrina, a representative of another snail family, the Planorbidae (Farag and El Sayad, 1995). This report of what is certainly predominantly a lymnaeid parasite from a snail of a different family is perhaps indicative of a rare host switch event. In the case of fasciolids, phylogenetic studies suggest that the primordial snail hosts for this particular group of digeneans were likely planorbids (Lotfy et al., 2008), so perhaps a lymnaeid-transmitted species like $F$. hepatica retains some ability to, at least occasionally, infect a snail of its ancestral host lineage?

\section{Evolutionary considerations}

Is the pressure imposed by digenean parasitism a factor that has favored the evolution of specificity in gastropod immune systems, and does this specificity in turn serve as a driver of digenean species diversity? As noted above, given the pervasive influence of digeneans in gastropod biology, it seems reasonable to think that digeneans have had a major impact on shaping the nature of gastropod immunity, including its degree of specificity. When an abundant, highly pathogenic and specialized lineage of parasites colonizes a particular group of hosts, we might expect the discriminatory powers of the hosts' immune system to become much more 
developed to recognize and deal with this parasite lineage than they might be with other lineages that do not pose as big a problem for them.

Given that digenean-snail specificity exists, one of the interesting paradoxes it poses, as exemplified by the schistosomes, is as follows. On the one hand, for any well-studied species of Schistosoma, it is evident that the snail host range is limited. On the other hand, from a comparison across Schistosoma species, it is also evident that the parasites have not cospeciated in any comprehensive way with the snail hosts: the S. japonicum species group is in pomatiopsid snails, the S. mansoni group is in Biomphalaria, the S. haematobium group in Bulinus, etc. So, given the snail host specificity shown by any extant schistosome species, and the difficulties it has in infecting non-host snails of different, species, genera or families, how is it that different Schistosoma species have been able to colonize quite different groups of snails? Host switches had to have occurred, albeit rarely, because it's almost certainly the case that Schistosoma diversified long after the snail lineages utilized came into being. Perhaps the liberalizing influence of facilitation mediated by other trematode species has permitted host switches to occur, particularly if the three-way interaction between schistosome, other trematode, and new snail host occurred over multiple generations such that the schistosome could adjust to the new host species without the need for help. This may in turn be accompanied by isolation from its relatives in the original snail host if accommodation to one immune system means failure in another. The point here is that the divergent immune systems between former and new hosts may be the fundamental driver that permits the emergence of a new digenean species. If the immune systems were more generalized, then one might imagine that host sharing would be very common, the resulting isolation between digenean species would be diminished, and the tendency to differentiate population of digeneans into distinctive species would be lost. Consider again the terrestrial styllomatophoran gastropods as discussed in section 2.1. If it is the case that as a group they suffer less burden of parasitism from digeneans, then one possible consequence of this might be that there is less diversified discriminatory power among different lineages in their immune 
systems, at least with respect to digeneans. As a consequence, the digeneans that do parasitize styllomatophorans may have broader host ranges and be less likely to eventually reveal cryptic species. In other words, digeneans have not driven immune specificity in this system, and this in turn has failed to generate great diversity among the digeneans involved.

In summary, we suggest that a pervasive feature of digenean-snail associations, their evident specificity, provides a unique opportunity to explore the specificity of gastropod immunobiology. Also, we argue that digeneans may have been a factor in driving the specificity in the first place, and that interactions among digenean species can facilitate host range expansions that can lead to host switches and parasite diversification. Finally we note that a number of modern studies of snail immunobiology have 1) provided some good candidate molecules that help explain the specificity, including production of both diverse parasite antigens and snail recognition molecules; 2) provided tangible ways to move forward to test some of the assertions made regarding the mechanistic basis of specificity; and 3) shed light on a system that might be particularly suited, given the abundance, impact and dependence of digeneans, to yield interesting features of invertebrate immunobiology.

Acknowledgments: This review would not be possible without past and present landmark contributions from many investigators in the field of comparative immunological studies of Biomphalaria snails. This study was supported by NIH grant 1P20RR18754, ESL acknowledges NIH grant AI101438 and a grant from the Bill and Melinda Gates Foundation through a Grand Challenges Explorations Initiative. 


\section{References}

Adema, C.M., Hanington, P.C., et al.,2010 Differential transcriptomic responses of Biomphalaria glabrata (Gastropoda, Mollusca) to bacteria and metazoan parasites, Schistosoma mansoni and Echinostoma paraensei (Digenea, Platyhelminthes). Molec. Immunol. 47, 849-860.

Adema C.M., Hertel, L.A., et al., 1997a. Infection with Echinostoma paraensei (Digenea) induces parasite-reactive polypeptides in the hemolymph of the gastropod host Biomphalaria glabrata. In: Beckage, N. (Ed) Parasite Effects on Host Physiology and Behavior, Chapman Press New York, pp 77-99.

Adema CM, Hertel LA, et al., 1997b. A family of fibrinogen-related proteins that precipitates parasite-derived molecules is produced by an invertebrate after infection. Proc. Nat. Acad. Sci. USA 94, 8691-8696.

Adema, C.M., Loker, E.S., 1997. Specificity and immunobiology of larval digenean-snail interactions. in Fried, B. and Graczyk, T.K., (Eds) Advances in trematode biology, CRC Press, Boca Raton, pp. 229-264.

Adema, C.M., van der Knaap, W.P.W., et al., 1991. Molluscan hemocyte-mediated cytotoxicity: the role of reactive oxygen intermediates. Rev. Aquat. Sci. 4, 201-223.

Barbosa, F.S., Barbosa, I., et al., 1959. Laboratory infections of the snail Planorbarius metidenjensis (Forbes) from French Morocco with a Brazilian strain of Schistosoma mansoni. Ann. Trop. Med. Parasitol. 53, 314-315.

Baron, O.L., van West, P., et al., 2013. Parental transfer of the antimicrobial protein LBP/BPI protects Biomphalaria glabrata eggs against oomycete infections. PLoS Pathog. 9(12): e1003792.

Basch, P.F.,1976. Intermediate host specificity in Schistosoma mansoni. Exp. Parasitol. 39, 150- 
169.

Bonner, K.M., Bayne, C.J., et al., 2012. Effects of $\mathrm{Cu} / \mathrm{Zn}$ superoxide dismutase (SOD1) genotype and genetic background on growth, reproduction and defense in Biomphalaria glabrata. PLoS Negl. Trop. Dis. 6, e1701.

Brown, D.S., 2005. Freshwater snails of Africa and their medical importance, second ed. Taylor and Francis Limited.

Buckley, K.M., Rast, J.P., 2012. Dynamic evolution of toll-like receptor multigene families in echinoderms. Front. Immunol. 5;3:136.

Butcher, A.R., Grove, D.I., 2001. Description of the life-cycle stages of Brachylaima cribbi n. sp (Digenea : Brachylaimidae) derived from eggs recovered from human faeces in Australia. Syst. Parasitol., 49, 211-221.

Caron, Y., Martens, K., et al., 2014, New insight in lymnaeid snails (Mollusca, Gastropoda) as intermediate hosts of Fasciola hepatica (Trematoda, Digenea) in Belgium and Luxembourg. Parasites \& Vectors 7:66

Chernin, E., Perlstein, J. M., 1971. Protection of snails against miracidia of Schistosoma mansoni by various aquatic invertebrates. J. Parasitol. 57, 217-219.

Christofi, T., Apidianakis. Y., 2013. Drosophila immune priming against Pseudomonas aeruginosa is short-lasting and depends on cellular and humoral immunity. F1000Res. 5;2:76.

Combes, C., Moné, H., 1987. Possible mechanisms of the decoy effect in Schistosoma mansoni transmission. Int. J. Parasitol. 17, 971-975.

Coustau, C., Yoshino, T.P., 2000. Flukes without snails: Advances in the in vitro cultivation of intramolluscan stages of trematodes. Expt. Parasitol. 94, 62-66.

Damian, R.T., 1964. Molecular mimicry - antigen sharing by parasite and host and its consequences. Am. Naturalist. 98, 129-149. 
Deleury, E., Dubreuil, G., et al., 2012. Specific versus non-specific immune responses in an invertebrate species evidenced by a comparative de novo sequencing study. PLoS One.7, e32512.

DeJong, R.J., Morgan, J.A.T., et al., 2001. Evolutionary relationships and biogeography of Biomphalaria (Gastropoda : Planorbidae) with implications regarding its role as host of the human bloodfluke, Schistosoma mansoni. Mol. Biol. Evol. 18, 2225-2239.

Donald, K.M., Kennedy, M., et al., 2004. Host specificity and molecular phylogeny of larval Digenea isolated from New Zealand and Australian topshells (Gastropoda : Trochidae). Int. J. Parasitol. 34, 557-568.

Erwin, D.H., Laflamme, M., et al., 2011. The Cambrian conundrum: early divergence and later ecological success in the early history of animals. Science 334, 1091-1097.

Esch, G.W., Barger M.A., et al., 2002. The transmission of digenetic trematodes: style, elegance, complexity. Integr. Comp. Biol.42: 304-312.

Farag, H.F., El Sayad, M.H., 1995. Biomphalaria alexandrina naturally infected with Fasciola hepatica. Trans. Roy Soc. Trp. Med. Hyg. 89: 36.

Ferrandon, D., Imler, J.L., et al., 2007. The Drosophila systemic immune response: sensing and signalling during bacterial and fungal infections. Nat. Rev. Immunol. 7, 862-874.

File, S.K., 1975. Superinfection of Biomphalaria glabrata with Schistosoma mansoni. J. Parasitol. $61,75-78$

Frandsen, F., 1979. Studies of the relationship between Schistosoma and their intermediate hosts. 3. Genus Biomphalaria and Schistosoma mansoni from Egypt, Kenya, Sudan, Uganda, West Indies (St Lucia) and Zaire (2 different strains - Katanga and Kinshasa). J. Helminthol. 53, 321-348.

Galinier,R., Portela, J., et al., 2013. Biomphalysin, a new $\beta$ pore-forming toxin involved in Biomphalaria glabrata immune defense against Schistosoma mansoni. PLoS Pathog. 9, 3, e1003216. 
Garcia, A.B., Pierce, R.J. et al. 2010. Involvement of the cytokine MIF in the snail host immune response to the parasite Schistosoma mansoni. PLoS Pathog. Sep 2010; 6(9): e1001115.

Ghosh, J., Lun, C.-M., et al., 2011. Invertebrate immune diversity. Dev. Comp. Immunol. 35, 959-974.

Guillou, F., Mitta, G., et al., 2004. Use of individual polymorphism to validate potential functional markers: case of a candidate lectin (BgSel) differentially expressed in susceptible and resistant strains of Biomphalaria glabrata. Comp. Biochem. Physiol. B Biochem. Mol. Biol., 138, 175-181.

Haas, W., 2003. Parasitic worms: strategies of host finding, recognition and invasion. German Zool. Soc. 106, 349-364.

Hahn, U.K., Bender, R.C., et al., 2001. Killing of Schistosoma mansoni sporocysts by hemocytes from resistant Biomphalaria glabrata: role of reactive oxygen species. J Parasitol. 87, 292299.

Hanelt, B., Lun, C.-M., et al., 2008. Comparative ORESTES-sampling of transcriptomes of immune-challenged Biomphalaria glabrata snails. J. Invert. Pathol. 99, 192-203.

Hanington, P.C., Lun, C.-M., et al., 2010a. Time series analysis of the transcriptional responses of Biomphalaria glabrata throughout the course of intramolluscan development of Schistosoma mansoni and Echinostoma paraensei. Int. J. of Parasitol. 40, 819-831.

Hanington, P.C.; Forys, M.A., et al., 2010b. Role for a somatically diversified lectin in resistance of an invertebrate to parasite infection. Proc. Natn. Acad. Sci. USA 107, 21087-21092.

Hanington, P.C., Forys, M.A., et al., 2012. A somatically diversified defense factor, FREP3, is a determinant of snail resistance to schistosome infection. PLoS Neglect. Dis. 6, e1591.

Hauton, C., Smith, V.J., 2007. Adaptive immunity in invertebrates: A straw house without a mechanistic foundation. BioEssays 29, 1138-1146.

Heyneman, D., 1966. Successful infection with larval echinostomes surgically implanted into the body cavity of the normal snail host. Expt. Parasitol. 18, 220-223. 
Hoffmann, J.A., Reichhart, J.M., 2002. Drosophila innate immunity: an evolutionary perspective. Nat. Immunol. 3, 121-126.

Johnson, P.T.J., Lund, P.J., et al., 2009. Community diversity reduces Schistosoma mansoni transmission, host pathology and human infection risk. Proc. Roy. Soc. B. Biol. Sci. 276, $1657-1663$.

Kagan, I.G, Geiger, S.J. 1965. The susceptibility of three strains of Australorbis glabratus to Schistosoma mansoni from Brazil and Puerto Rico. J. Parasitol. 51, 622-627.

Kawasaki, M., Delamare-Deboutteville, et al., 2013. Hemiuroid trematode sporocysts are undetected by hemocytes of their intermediate host, the ark cockle Anadara trapezia: Potential role of surface carbohydrates in successful parasitism. Fish Shellfish Immunol. 35, 1937-1947.

Klein, J., 1989. Are Invertebrates Capable of Anticipatory Immune Responses? Scand. J. Immunol. 29, 499-505.

Kechemir, N., Combes, C., 1982. Development of Schistosoma haematobium in Planorbarius metidjensis by means of microsurgical transplantations. C.R. Séances Acad. Sci. III. 295, $505-508$.

Kraaijeveld, A.R., Layen, et al., 2012. Lack of phenotypic and evolutionary cross-resistance against parasitoids and pathogens in Drosophila melanogaster. PLoS One. 7, e53002.

Lehr, T., Frank, S., et al., 2010. N-Glycosylation patterns of hemolymph glycoproteins from Biomphalaria glabrata strains expressing different susceptibility to Schistosoma mansoni infection. Expt. Parasitol. 126, 592-602.

Lie, K.J., 1982. Survival of Schistosoma mansoni and other trematode larvae in the snail Biomphalaria glabrata - A discussion of the interference theory. Trop. Geogr. Med. 34, 111122.

Lie, K.J., Heyneman. D., 1975. Studies on resistance in snails: a specific tissue reaction to Echinostoma lindoense in Biomphalaria glabrata snails Int. J. Parasitol. 5,621-625. 
Lie, K.J., Heyneman. D., 1976. Studies on resistance in snails. 3. Tissue reactions to Echinostoma lindoense sporocysts in sensitized and resensitized Biomphalaria glabrata. J. Parasitol. 62, $51-58$.

Lie, K.J., Heyneman, D., 1979. Acquired resistance to echinostomes in 4 Biomphalaria glabrata strains. Int. J. Parasitol. 9, 533-537.

Lie, K.J., Heyneman. D., et al., 1976. Studies on resistance in snails. 4. Induction of ventricular capsules and changes in the amebocyte-producing organ during sensitization of Biomphalaria glabrata snails. J. Parasitol. 62, 286-291.

Lie, K.J., Heyneman. D., et al.,1975. Studies on resistance in snails: specific resistance induced by irradiated miracidia of Echinostoma lindoense in Biomphalaria glabrata snails. Int. J. Parasitol. 5, 627-631.

Lie, K.J., Jeong, K.H., et al.,1983. Acquired resistance in snails. Induction of resistance to Schistosoma mansoni in Biomphalaria glabrata. Int. J. Parasitol. 13, 301-304.

Lie, K.J., Jeong, K.H., et al.,1987. Molluscan host reactions to helminthic infection. in: Soulsby, E.J.L. (Ed) Immune responses in parasitic infections: immunology, immunopathology, and immunoprophylaxis. Volume IV: Protozoa, arthropods, and invertebrates. CRC Press, Boca Raton Florida pp. 211-270

Lockyer A.E., Emery, A.M., et al., 2012, Early differential gene expression in haemocytes from resistant and susceptible Biomphalaria glabrata strains in response to Schistosoma mansoni. PLoS One.;7(12):e51102.

Lockyer, A.E., Spinks, J.N., et al., 2007. Biomphalaria glabrata transcriptome: identification of cell-signalling, transcriptional control and immune-related genes from open reading frame expressed sequence tags (ORESTES). Dev. Comp Immunol., 31, 763-782.

Lockyer AE, Spinks J, et al., 2008. Biomphalaria glabrata transcriptome: cDNA microarray profiling identifies resistant- and susceptible-specific gene expression in haemocytes from snail strains exposed to Schistosoma mansoni.BMC Genomics. 9:634. 
Loker, E.S., Adema, C.M., et al.,2004. Invertebrate immune systems - not homogeneous, not simple, not well understood. Immunol. Rev.198, 10-24.

Loker, E.S., Bayne, C.J., 1982. In vitro encounters between Schistosoma mansoni primary sporocysts and hemolymph components of susceptible and resistant strains of Biomphalaria glabrata. Am. J. Trop. Med. Hyg. 31, 999-1005.

Loker, E.S., Cimino, D.F., et al.,1992. Excretory-secretory products of Echinostoma paraensei sporocysts mediate interference with Biomphalaria glabrata hemocyte functions. J. Parasitol. $78,104-115$.

Lotfy, W.M., Brant, S.V., et al.,2008. Evolutionary origins, diversification and biogeography of liver flukes (Digenea, Fasciolidae). Am. J. Trop. Med. Hyg. 79, 248-255.

Maldonado, A., Vieira, G.O., et al., 2001. Biological aspects of a new isolate of Echinostoma paraensei (Trematoda : Echinostomatidae): susceptibility of sympatric snails and the natural vertebrate host. Parasitol. Res., 87, 853-859.

Manga-Gonzalez, M.Y., Gonzalez-Lanza, C., et al., 2001. Contributions to and review of dicrocoeliosis, with special reference to the intermediate hosts of Dicrocoelium dendriticum. Parasitology 123, S91-S114.

McKindsey, C.W., McLaughlin, J.D., 1995. Species-specific and size-specific infection of snails by Cyclocoelum mutabile (Digenea, Cyclocoelidae). J. Parasitol. 81, 513-519.

Minchella, D.J., Sollenberger, K.M., et al., 1995. Distribution of schistosome genetic diversity within molluscan intermediate hosts. Parasitology 111, 217-220.

Mitta, G., Adema, C.M., et al., 2012. Compatibility polymorphism in snail/schistosome interactions: From field to theory to molecular mechanisms. Dev. Comp. Immunol. 37, 1-8.

Mitta, G., Galinier, R., et al., 2005. Gene discovery and expression analysis of immune-relevant genes from Biomphalaria glabrata hemocytes. Dev. Comp. Immunol., 29, 393-407.

Moné, Y., Gourbal, B., et al., 2010. A large repertoire of parasite epitopes matched by a large 
repertoire of host immune receptors in an invertebrate host/parasite model. PLoS Neglect. Trop. Dis. 4, e813.

Moné, Y., Ribou, A.C., et al., 2011. An example of molecular co-evolution: reactive oxygen species (ROS) and ROS scavenger levels in Schistosoma mansoni/Biomphalaria glabrata interactions. Int. J. Parasitol. 41, 721-730.

Nunez, P. E., De Jong-Brink, M. 1997. The suppressive excretory-secretory product of Trichobilharzia ocellata: A possible factor determining compatibility in parasite-host interactions. Parasitology 115, 193-203.

Petney, T.N., Andrews, R.H., et al., 2013. The zoonotic, fish-borne liver flukes Clonorchis sinensis, Opisthorchis felineus and Opisthorchis viverrini. Int. J. Parasitol. 43, 1031-1046.

Pham, L.N., Dionne, M.S., et al., 2007. A specific primed immune response in Drosophila is dependent on phagocytes. PLoS Pathog. 3,3, e26.

Portela, J., Duval, D., et al.,2013. Evidence for specific genotype-dependent immune priming in the lophotrochozoan Biomphalaria glabrata snail. J. Innate Immunity 5, 261-276.

Raghavan., N, Miller, A.N., et al., 2003.Comparative gene analysis of Biomphalaria glabrata hemocytes pre- and post-exposure to miracidia of Schistosoma mansoni. Mol. Biochem. Parasitol., 126, 181-191.

Renwrantz, L., 1986. Lectins in molluscs and arthropods: their occurrence, origin and roles in immunity. Proc. Zool. Soc. Lond. 56, 81-93.

Richards, C.S., Knight, M., et al., 1992. Genetics of Biomphalaria glabrata and its effect on the outcome of Schistosoma mansoni infection. Parasitol. Today 8, 171-174.

Rollinson, D., Stothard, J.R., et al., 2001. Interactions between intermediate snail hosts of the genus Bulinus and schistosomes of the Schistosoma haematobium group. Parasitology 123, S245-S260.

Sapp, K.K., Loker, E.S., 2000a. Mechanisms underlying digenean - snail specificity: role of miracidial attachment and host plasma factors. J. Parasitol. 86, 1012 - 1019. 
Sapp, K.K., Loker, E.S., 2000b. A comparative study of mechanisms underlying digenean - snail specificity: in vitro interactions between hemocytes and digenean larvae. J. Parasitol. 86, $1020-1029$.

Sire, C., Rognon, A., Theron, A., 1998. Failure of Schistosoma mansoni to reinfect Biomphalaria glabrata snails: acquired humoral resistance or intra-specific larval antagonism? Parasitology $117,117-122$.

Southgate, V.R., Brown, D.S., et al.,1989. The influence of Calicophoron microbothrium on the susceptibility of Bulinus tropicus to Schistosoma bovis. Parasitol. Res. 75, 381-391.

Spatz, L., Gonzalez Cappa, S.M., et al., 2012. Susceptibility of wild populations of Biomphalaria spp. from Neotropical South America to Schistosoma mansoni and interference of Zygocotyle lunata. J. Parasitol. 98, 1291-1295.

Steinauer, M.L., Mwangi, I.N., et al., 2008. Interactions between Natural Populations of Human and Rodent Schistosomes in the Lake Victoria Region of Kenya: A Molecular Epidemiological Approach. PLoS Negl. Trop. Dis. 2, 4, e222

Steinauer, M.L., Hanelt, B., et al. 2009. Genetic structure of Schistosoma mansoni in western Kenya: the effects of geography and host sharing. Int. J. Parasitol. 39, 1353-1362.

Stout, B.A., Adema, C.M., et al., 2008. The biology of FREPs: diversified lectins with fibrinogen-related domains from the freshwater snail Biomphalaria glabrata. in: Vasta G., Ahmed H. (Eds) Animal Lectins, A Functional View. CRC Press Taylor \& Francis, pp 475491.

Sudds Jr., R.H., 1960. Observations of schistosome miracidial behavior in the presence of normal and abnormal snail hosts and subsequent tissue studies of these hosts. J. Elisha Mitchell Sci. Soc. $76,121-133$.

Sullivan, J.T., Yeung, J.T., 2011. Incompatibility between miracidia of Schistosoma mansoni and Helisoma duryi occurs at two stages: penetration and intramolluscan establishment. J. Parasitol. 97, 743-745. 
Théron, A., Coustau, C.. 2005. Are Biomphalaria snails resistant to Schistosoma mansoni? J. Helminthol., 79, 187-191.

Van der Knaap, W.P.W., Loker, E.S., 1990. Immune mechanisms in trematode-snail interactions. Parasitol. Today.6, 175-182.

Vasquez, R.E., Sullivan, J.T., 2001. Further characterization of passively transferred resistance to Schistosoma mansoni in the snail intermediate host Biomphalaria glabrata. J. Parasitol.87, $1360-1365$.

Vasta, G.R., Ahmed, H., et al., 2012. Galectins as self/non-self recognition receptors in innate and adaptive immunity: an unresolved paradox. Front. Immunol. 3,199.

Walker, J.C., 1979. Austrobilharzia terrigalensis - schistosome dominant in interspecific interactions in the molluscan host. Int. J. Parasitol. 9, 137-140.

Yoshino, T.P., Dinguirard, N., et al., 2008. Molecular and functional characterization of a tandem-repeat galectin from the freshwater snail Biomphalaria glabrata, intermediate host of the human blood fluke Schistosoma mansoni. Gene 411, 46-58.

Yoshino, T.P., Wu, X-J., et al., 2012. Glycotope sharing between snail hemolymph and larval schistosomes: larval transformation products alter shared glycan patterns of plasma proteins. PLoS Neglect. Trop. Dis. 6, Article Number: e1569.

Yoshino, T.P., Wu, X-J., et al.,2013. Circulating Biomphalaria glabrata hemocyte subpopulations possess shared schistosome glycans and receptors capable of binding larval glycoconjugates. Expt. Parasitol. 133, 28-36.

Zhang, S.M., Adema, C.M., et al., 2004. Diversification of Ig superfamily genes in an invertebrate. Science 305, 251-254.

Zhang, S.M., Zeng, Y., et al.,2008. Expression profiling and binding properties of fibrinogenrelated proteins (FREPs), plasma proteins from the schistosome snail host Biomphalaria glabrata. Innate Immun., 14, 175-189. 